\title{
Head to head comparisons of two modalities of perfusion adenosine stress echocardiography with simultaneous SPECT Petri Gudmundsson ${ }^{1}$, Kambiz Shahgaldi², Reidar Winter ${ }^{2,3}$, Magnus Dencker*4, Mariusz Kitlinski ${ }^{5}$, Ola Thorsson ${ }^{4}$, Lennart Ljunggren ${ }^{1}$ and Ronnie B Willenheimer6,7
}

Address: ${ }^{1}$ Department of Biomedical Laboratory Science, Malmö University, Malmö, Sweden, ${ }^{2}$ Department of Cardiology, Karolinska University Hospital Huddinge, Stockholm, Sweden, ${ }^{3}$ Department of Clinical Physiology, Karolinska University Hospital Huddinge, Stockholm, Sweden, ${ }^{4}$ Department of Clinical Physiology, Lund University, Malmö University Hospital, Malmö, Sweden, ${ }^{5}$ Department of Cardiology, Lund University, Malmö University Hospital, Malmö, Sweden, ${ }^{6}$ Department of Clinical Sciences, Medicine/Cardiology, Lund University, Malmö University Hospital, Malmö, Sweden and ${ }^{7}$ Heart Health Group, Malmö, Sweden

Email: Petri Gudmundsson - petri.gudmundsson@mah.se; Kambiz Shahgaldi - kambiz.shahgaldi@karolinska.se;

ReidarWinter - reidar.winter@karolinska.se; Magnus Dencker* - magnus.dencker@skane.se; Mariusz Kitlinski - mariusz.kitlinski@skane.se; Ola Thorsson - ola.thorsson@skane.se; Lennart Ljunggren - lennart.ljunggren@mah.se;

Ronnie B Willenheimer - ronnie.willenheimer@med.lu.se

* Corresponding author

Published: 20 April 2009

Cardiovascular Ultrasound 2009, 7:19 doi:10.1186/1476-7/20-7-19
Received: 10 March 2009

Accepted: 20 April 2009

This article is available from: http://www.cardiovascularultrasound.com/content/7/1/19

(c) 2009 Gudmundsson et al; licensee BioMed Central Ltd.

This is an Open Access article distributed under the terms of the Creative Commons Attribution License (http://creativecommons.org/licenses/by/2.0), which permits unrestricted use, distribution, and reproduction in any medium, provided the original work is properly cited.

\begin{abstract}
Background: Real-time perfusion (RTP) contrast echocardiography can be used during adenosine stress echocardiography (ASE) to evaluate myocardial ischemia. We compared two different types of RTP power modulation techniques, angiomode (AM) and high-resolution grayscale (HR), with 99mTc-tetrofosmin single-photon emission computed tomography (SPECT) for the detection of myocardial ischemia.
\end{abstract}

Methods: Patients with known or suspected coronary artery disease (CAD), admitted to SPECT, were prospectively invited to participate. Patients underwent RTP imaging (SONOS 5500) using AM and HR during Sonovue ${ }^{\circledR}$ infusion, before and throughout the adenosine stress, also used for SPECT. Analysis of myocardial perfusion and wall motion by RTP-ASE were done for AM and HR at different time points, blinded to one another and to SPECT. Each segment was attributed to one of the three main coronary vessel areas of interest.

Results: In 50 patients, 150 coronary areas were analyzed by SPECT and RTP-ASE AM and HR. SPECT showed evidence of ischemia in 13 out of 50 patients. There was no significant difference between $A M$ and $H R$ in detecting ischemia $(p=0.08)$. The agreement for $A M$ and $H R$, compared to SPECT, was $93 \%$ and $96 \%$, with Kappa values of 0.67 and 0.75 , respectively $(p<0.00 \mathrm{I})$.

Conclusion: There was no significant difference between AM and HR in correctly detecting myocardial ischemia as judged by SPECT. This suggests that different types of RTP modalities give comparable data during RTP-ASE in patients with known or suspected CAD. 


\section{Background}

Patients with suspected myocardial ischemia are often assessed using different types of exercise tests for ischemic evaluation, according to current clinical guidelines $[1,2]$. Exercise ECG is considered the first-line technique for assessment of ischaemia, whereas single-photon emission computed tomography (SPECT) or dobutamine atropine stress echocardiography (DSE) are suggested when exercise ECG are non-diagnostic or non-interpretable $[3,4]$. Both SPECT and DSE are more accurate methods than exercise ECG, although more expensive [4-7].

Adenosine stress echocardiography (ASE) is a less expensive technique as compared to SPECT and more tolerable compared to DSE for the assessment of patients with suspected coronary artery disease. However, ASE, using only wall motion evaluation, is less accurate for the detection of myocardial ischemia as compared to DSE and SPECT. [8-10]. The use of second-generation contrast agents in myocardial contrast echocardiography enables assessment of myocardial perfusion during ECG triggered image acquisition echocardiography with high mechanical index, i.e. harmonic power Doppler. This technique is not only technically demanding, but also has limitations, e.g. because no wall motion evaluation is possible.

Real-time myocardial perfusion echocardiography (RTP) has recently been developed using highly contrast specific, low mechanical index imaging techniques. RTP during intravenous infusion of a second-generation contrast agent allows for simultaneous analysis of myocardial perfusion and wall motion. Earlier studies with RTP have shown promising results for the evaluation of myocardial perfusion [11-18]. However, there is limited clinical data on the accuracy of RTP-ASE for the detection of myocardial ischemia in unselected patient groups. The RTP techniques continue to evolve and new improvements are developed continuously. There are, however, only few studies carried out to find out if the new developments really improve the evaluation of ischemia.

Adenosine is a sub-optimal stressor for wall motion analysis and may be less sensitive in detecting ischemia [3]. On the other hand, perfusion defects seem to be more visible with adenosine compared to dobutamine [9]. The myocardial contrast echocardiography technique is still burdened with perfusion artifacts and echocardiographic shadowing, which limit the number of interpretable myocardial segments. These segments can in most cases, using RTP, still be interpreted with wall motion analysis, which therefore increases the usefulness of RTP-ASE without diminishing accuracy considerably [15]. Thus, combining perfusion and wall motion assessment, RTP-ASE has the potential of being as accurate and feasible as DSE and SPECT, and may be a swift, bedside-accessible, useful decision-making tool for risk assessment of patients with suspected myocardial ischemia.

Two techniques of RTP power modulation have been developed. The first technique available was angio-mode (AM), in which echoes from contrast bubbles are displayed as colored pixels in the two dimensional images and are, therefore, easily differentiated from the tissue echoes, which are displayed in grey scale. The most recently developed power modulation technique is the high-resolution grey scale (HR). Using HR, the only echoes displayed are grey scale echoes from contrast bubbles. The HR technique is meant to suppress all echoes originated from tissue and has a higher spatial resolution compared to AM. The difference between the two techniques has to our knowledge not been examined by head to head comparison.

The aim of the present study was to compare the two power modulation techniques, AM and HR, during RTPASE, for the detection of myocardial ischemia, as judged by $99 \mathrm{mTc}$-tetrofosmin SPECT, in a clinical patient population with known or suspected myocardial ischemia.

\section{Methods \\ Patient population}

The patient population consisted of 51 randomly selected patients with known or suspected coronary artery disease, admitted to SPECT evaluation, to participate in the study. The patient's acoustic windows were not screened prior to inclusion. One of the included patients had non-interpretable echocardiography images, both regarding wall motion and perfusion, and was therefore excluded from the study. The institutional ethics committee of the Lund University, Sweden, approved the study (Lu 113-03). Written informed consent was obtained from all participating patients.

\section{Study protocol}

\section{Myocardial contrast echocardiography}

The echocardiographic equipment used was a Sonos 5500 (Philips, Andover, MA, USA) with S3 probe and RTP using power modulation AM and HR. Patients were examined in a left lateral recumbent position. The second-generation contrast agent Sonovue ${ }^{\circledast}$ was infused in the left decubital vein using an infusion pump dedicated for this purpose (VueJect ${ }^{\oplus}$ Esaote, Genova, Italy; Bracco ${ }^{\mathrm{TM}}$, Milano, Italy), which automatically rotates the syringe to prevent sedimentation. The infusion rate of Sonovue ${ }^{\circledast}$ was set between 1.0 and $1.3 \mathrm{ml} / \mathrm{min}$ [19]. Adenosine and echo contrast were infused in the same peripheral venous catheter, using a separate infusion pump through a three-way stopcock. Adenosine was given at an infusion rate of 100 $\mu \mathrm{g} / \mathrm{kg} / \mathrm{min}$ during one minute, and was thereafter increased to $140 \mu \mathrm{g} / \mathrm{kg} / \mathrm{min}$. 
All 50 patients underwent RTP imaging (mechanical index $=0.1$ ) during infusion of echo contrast, at rest and after a minimum of one minute of hyperemia during adenosine stress (at $140 \mu \mathrm{g} / \mathrm{kg} / \mathrm{min}$ ). Image acquisition was started after a minimum time of one minute of Sonovue ${ }^{\circledast}$ infusion. RTP image loops containing 8-10 heartbeats were collected from the parasternal long- and short-axis and apical fourand two-chamber views, respectively. At the beginning of each loop a destruction impulse of 10 high mechanical index frames (mechanical index $=1.5$ ) were given to destroy all contrast micro bubbles in the myocardium [20].

During RTP AM, the angio-mode gain was set at between 60 and $70 \%$, depending on what was suitable for the individual patient as judged by a visual on-line assessment, and 2D grayscale gain was set at zero. During RTP HR the grey-scale gain was set between 90 and 95\%, depending on what was suitable for the individual patient, as judged by a visual on-line assessment. Focus was set close to the base of the left ventricle. All images were stored digitally for later off-line analysis.

\section{SPECT}

The rest and stress studies were performed using a 2-day protocol, starting with injection of $600 \mathrm{MBq} 99 \mathrm{mTC}$-tetrofosmin at stress. Stress was simultaneous with the RTPASE. Normal findings at stress were not followed by a rest study $[21,22]$. Pathological stress studies were followed by a rest study with injection of $800 \mathrm{MBq}{ }^{99 m}$ Tc-tetrofosmin. A five-minute adenosine infusion protocol was used. Patients who had cardiac medications, which could interfere with the stress test, were informed to have their medication interrupted prior to the stress test. The decision whether to interrupt the drug administration was at the discretion of the referring physician. Starting the infusion with $100 \mu \mathrm{g} / \mathrm{ml} / \mathrm{min}$ of adenosine for 1 minute, the dose was then increased to $140 \mu \mathrm{g} / \mathrm{ml} / \mathrm{min}$ for two minutes before injecting ${ }^{99 \mathrm{~m} T c-t e t r o f o s m i n}$. Infusion of adenosine was continued for $2 \mathrm{~min}$ after the injection of ${ }^{9 \mathrm{~m} T \mathrm{Tc}-\text { tetro- }}$ fosmin. The scintigraphic data were acquired one hour after the end of the stress test, using continuous SPECT over 180 degree elliptical rotation from the 45 degree right anterior oblique position, with a dual-head gamma camera (Siemens AG Medical Solutions, Erlangen, Germany). Low energy high-resolution collimator and a zoom factor of 1.0 were used. We obtained 64 projections in a $128 \times 128$ matrix, with an acquisition time of $20 \mathrm{~s}$ per projection. Tomographic reconstruction and calculation of short axis slice images were performed using Siemens software. A two-dimensional Butterworth pre-reconstruction filter was used with critical frequency of 0.35 , order 5. For each patient, the same sets of short axis slices were then processed with an automatic software package (4DMSPECT) on a Siemens e.soft workstation. The software package defined apex and base and generated, coronal, longitudinal, sagital tomographic slices as well as polar maps with schematic map of the territories of the main coronary arteries used for scoring. Radiotracer uptake of the vascular segments was scored visually and stress images were compared with rest images regarding ischemia and infarct. The specialist in nuclear medicine who performed the scoring was blinded to the results of the RTP analysis.

\section{RTP-ASE image interpretation}

Image interpretation was performed off line, analyzing myocardial perfusion and wall motion by RTP-ASE, using the EnConcert Image Diagnosis Application (Philips, Andover, MA, USA). A separate analysis exclusively of perfusion was also made for both AM and HR to estimate the value of sole perfusion analysis. AM and HR were evaluated on separate occasions blinded to one another and blinded to the result of the SPECT interpretation.

Each segment was attributed to one of the three main coronary vessel areas of interest; the left anterior descending (LAD); the left circumflex (LCx); and the right posterior descending (RPD) (see Figure 1). Myocardial ischemia was visually evaluated comparing rest and stress images, using both perfusion and wall motion analysis in a complementary manner. A visually detected perfusion defect during stress was used as the principal marker of ischemia. Thus, a myocardial segment was considered ischemic if perfusion was impaired in the stress images, compared to the rest images [9]. Perfusion defects were analyzed at the earliest four beats following the destruction impulse at rest and after two beats at peak stress.

Wall motion was used in addition to reveal perfusion defect artifacts at rest and to evaluate segments with suspected perfusion artifacts at stress. Since wall motion should not be normal if a segment has a true perfusion defect at rest, a perfusion defect at rest was considered to be an artifact when wall motion was normal in that segment. A perfusion defect at peak stress was considered to be an artifact if there was a suspicion of a perfusion artifact, such as lateral or anterior shadowing from ribs or lungs, or basal segments shadowed by contrast. In such segments, the ischemic evaluation was based on wall motion analysis alone. If wall motion decreased at stress compared to rest images, the segment was considered ischemic. Since perfusion can be decreased without a decrease in wall motion in ASE, the use of solitary wall motion analysis in segments with perfusion artifacts might decrease the sensitivity with regard to ischemia. However, this complementary use of wall motion analysis increases the number of interpretable segments without negatively affecting specificity [11].

\section{Statistical analysis}

Power calculation for the RTP-ASE comparison to SPECT was based on a sensitivity and specificity between 80 and 


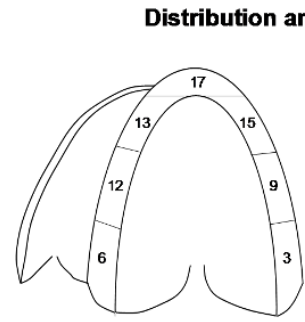

LAD: $12,13,15,17$

LCX: 9,3

RCA: 6

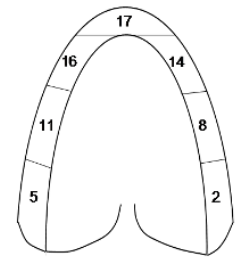

LAD: $2,8,14,16,17$

RCA: 5,11

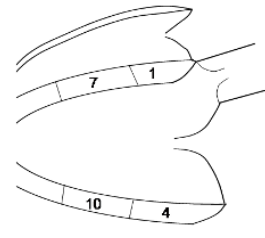

LAD: 1,7

LCX: 4, 10
Figure I

Distribution territories of the three main coronary arteries. Distribution territories of the three main coronary arteries in a 17 segment model. Left anterior descending (LAD), left circumflex (LCx) and right coronary artery (RCA).

$90 \%$ of the methods used. We assumed a sensitivity and a specificity of $85 \%$ in the study. With 50 patients we would have a $95 \%$ confidence interval of $\pm 10 \%$ around sensitivity and specificity. The SPSS ${ }^{\circledast}$ (Version 12.0.1, Chicago, IL, USA) statistical program was used for the statistical analysis. We calculated sensitivity and specificity, positive and negative predictive values (PPV, NPV), as well as accuracy and Kappa values in the three predefined distribution areas of the three main coronary vessels. To assess differences between AM and HR, the chi-squared test was performed. Method of reference for the ischemia evaluation in the study was the presence or absence of reversible ischemia in the SPECT examination. Results are expressed as mean $\pm \mathrm{SD}$ and as percent. $\mathrm{P}<0.05$ denoted significance.

\section{Results}

Baseline characteristics including clinical data extracted from patient's records are shown in Table 1 . Mean age of the 50 patients was 70 years, two thirds were women and mean left ventricular ejection fraction was close to normal. A history of previous myocardial infarction was found in less than half of the patients, around one third had undergone previous coronary intervention, a majority had no previous hospitalization or intervention due to coronary artery disease, and around one fifth of the patients had no cardiac medication. At SPECT, 13 (26\%) of the patients were ischemic in at least one coronary area.

Of 150 coronary areas assessed, all were considered interpretable and were analyzed both using SPECT and RTP with AM and HR. The overall level of agreement between RTP-ASE and SPECT in detecting ischemia was 93\% for AM and $96 \%$ for HR. The chi-square test for difference between AM and HR in correctly judging ischemia was borderline-significant ( $\mathrm{p}=0.08$ ). The Kappa values were 0.67 for AM and 0.75 for HR $(\mathrm{p}<0.001)$. Accuracy, sensitivity, specificity, predictive values and Kappa values for the detection of myocardial ischemia of RTP-ASE AM and $\mathrm{HR}$, in the respective coronary areas, compared to SPECT are shown in Table 2. HR generally showed somewhat higher values for kappa, accuracy, positive prediction and specificity, whereas AM showed higher sensitivity values. The NPVs were similar. The same comparative values for the sole perfusion interpretation concerning AM and HR compared to SPECT are displayed in Table 3, demonstrating differences similar to the combined perfusion and wall motion analysis. Accuracy and Kappa values for the agreement between AM and HR are shown in Table 4, indicating lesser agreement between AM and HR than for their respective comparison with SPECT. Figure 2 represents a graph of accuracy between different modalities of RTP-ASE versus SPECT.

In Table 5, the numbers of non-interpretable coronary areas are presented. In the sole perfusion analysis the numbers include coronary areas considered to be perfusion artifacts. There is evidence of similar loss of interpretable coronary areas for both AM and HR.

Inter- and intra-observer agreements for the interpretation of ischemia according to the RTP-ASE AM and HR examinations are presented in Table 6.

A movie of RTP-ASE images of a perfusion defect at stress is presented [see Additional file 1].

\section{Discussion}

The results from the present study show overall similarities, but some differences, although no statistically significant, between the two different types of RTP power modulation techniques, thus data derived from different techniques for RTP-ASE are reasonably interchangeable. As judged by SPECT, there were highly significant agreements for both AM and HR in detecting ischemia. The finding that RTP can be used to accurately detect ischemia is in line with previous investigations $[11-15,17,18]$. Although there were no significant differences between $\mathrm{AM}$ and HR in agreement, there were some differences worth discussing.

Both methods showed high NPVs, which is of importance for correctly acquitting ischemia in patients. The NPV is especially important in this study cohort, since the prevalence of reversible ischemia was quite high (26\%). Consequently, the value of PPV becomes relatively less important in this study. Wall motion abnormalities were present in $60 \%$ of the patients, indicating a very high prevalence of ischemic heart disease. However, the objective was to assess the accurate detection of reversible ischemia 
Table I: Patients characteristics, including clinical data extracted from patient's records.

\begin{tabular}{lc}
\hline Age & $70( \pm 8)$ \\
Male & $36 \%$ \\
LVEF at rest & $55( \pm 9) \%$ \\
Previous AMI & $40 \%$ \\
Previous PCI & $22 \%$ \\
Previous CABG & $16 \%$ \\
Heart failure & $12 \%$ \\
Hypertension & $54 \%$ \\
Valvular surgery & $0 \%$ \\
Beta-blocker & $54 \%$ \\
ACE inhibitor & $28 \%$ \\
ARB & $14 \%$ \\
Nitro-glycerin (short acting) & $60 \%$ \\
Nitrates (long acting) & $28 \%$ \\
Diuretics & $29 \%$ \\
Calcium blocker & $24 \%$ \\
Sinus rhythm & $92 \%$ \\
Dilated left ventricle & $10 \%$ \\
Dilated left atrium $(n=29)$ & $31 \%$ \\
Significant valvular disease $(n=31)$ & $6 \%$ \\
Regional WMA/PD at rest & $60 \%$ \\
\hline
\end{tabular}

LVEF, left ventricular ejection fraction; AMI, acute myocardial infarction; $\mathrm{PCl}$, percutaneous coronary intervention, $\mathrm{CABG}$, coronary artery bypass grafting; $A C E$, angiotensin converting enzyme; ARB, angiotensin-receptor blocker, WMA, wall motion abnormality; PD, perfusion defect.

using AM and HR respectively, not to reveal the presence or absence of ischemic heart disease. Hence, any difference between AM and HR regarding PPVs becomes less important. Using SPECT as reference, HR showed higher values of kappa and accuracy, suggesting that $\mathrm{HR}$ is better suited than AM in this kind of population, i.e. with high pre-test probability of reversible myocardial ischemia. On the other hand, AM seems to have somewhat slightly higher sensitivity, which means that the risk of a false neg- ative test values could actually be higher for HR. Sensitivity and specificity are somewhat connected to PPV and $\mathrm{NPV}$, respectively, but are less affected by the prevalence of ischemia in the actual patient sample examined. Sensitivity and specificity values may, therefore, provide more reliable information about the precision of the test, rather than about the health of the patients. The finding that sensitivity figures were higher for AM than for HR indicates that AM RTP-ASE may be better at detecting the true reversibly ischemic patient, while the higher specificity values for HR might represent better identification of nonischemic patients, as judged by SPECT. The slightly better sensitivity for AM might be due to the potentially easier visual assessment, to some point since echoes from contrast bubbles are displayed as colored pixels, in distinction to HR, where echoes are displayed in grey scale, but probably to a greater extent due to the lower resolution in AM. The lower resolution enables possible higher sensitivity because larger pixels provide more echo signal per pixel to process (higher statistical power) and thus a enhanced signal-to-noise ratio.

The higher kappa and accuracy values could therefore be due to superiority in correctly detecting non-ischemic territories, which were predominating in the present study population. The similarities and differences between AM and HR were approximately similar in the sole perfusion analysis, as compared with the combined perfusion and wall motion analysis. This indicates that agreement with SPECT can be maintained using wall motion analysis in coronary areas with perfusion artifacts, as previously suggested [15]. This should be viewed in relation to the results of non-interpretable coronary areas; where the high number of non-interpretable coronary areas in the sole perfusion analysis clearly illustrates lower feasibility, compared to the combined perfusion and wall motion

Table 2: RTP-ASE angio-mode (AM) versus high resolution (HR) with combined perfusion and wall motion analysis.

\begin{tabular}{|c|c|c|c|c|c|c|}
\hline & & $\begin{array}{c}\text { All CA } \\
(n=150)\end{array}$ & $\begin{array}{l}\text { Patient } \\
(\mathrm{n}=50)\end{array}$ & $\begin{array}{c}\text { LAD } \\
(n=50)\end{array}$ & $\begin{array}{c}\text { LCx } \\
(n=50)\end{array}$ & $\begin{array}{c}\text { RPD } \\
(n=50)\end{array}$ \\
\hline AM & Accuracy (\%) & 93 & 88 & 88 & 94 & 98 \\
\hline AM & PPV (\%) & 57 & 71 & 54 & 50 & 100 \\
\hline AM & NPV (\%) & 99 & 97 & 100 & 100 & 98 \\
\hline AM & Sensitivity (\%) & 92 & 92 & 100 & 100 & 75 \\
\hline AM & Specificity (\%) & 93 & 87 & 86 & 94 & 100 \\
\hline AM & Kappa & $0.67 * * *$ & $0.72 * * *$ & $0.63 * * *$ & $0.64 * * *$ & $0.85^{* * * *}$ \\
\hline HR & Accuracy (\%) & 96 & 92 & 90 & 96 & 100 \\
\hline HR & PPV (\%) & 77 & 91 & 67 & 67 & 100 \\
\hline HR & NPV (\%) & 98 & 92 & 93 & 98 & 100 \\
\hline HR & Sensitivity (\%) & 77 & 78 & 57 & 67 & 100 \\
\hline HR & Specificity (\%) & 98 & 97 & 95 & 98 & 100 \\
\hline HR & Kappa & $0.75 * * *$ & $0.78^{* * * *}$ & $0.56 * * *$ & $0.65^{* * * *}$ & $1.00 * * *$ \\
\hline
\end{tabular}

Accuracy, positive (PPV) and negative (NPV) predictive values, sensitivity, specificity and Kappa of RTP-ASE using SPECT as method of reference. $C A$, coronary area; LAD, left anterior descending coronary artery; LCx, left circumflex artery; RPD, right posterior descending coronary artery, *** $=p<0.001$. 
Table 3: RTP-ASE angio-mode (AM) versus high resolution (HR) using solitary perfusion analysis.

\begin{tabular}{|c|c|c|c|c|c|c|}
\hline & & $\begin{array}{c}\text { Any CA } \\
(\mathrm{n}=134)\end{array}$ & $\begin{array}{l}\text { Patient } \\
(\mathrm{n}=39)\end{array}$ & $\begin{array}{c}\text { LAD } \\
(n=44)\end{array}$ & $\begin{array}{c}\text { LCx } \\
(n=42)\end{array}$ & $\begin{array}{c}\text { RPD } \\
(n=48)\end{array}$ \\
\hline AM & Accuracy (\%) & 89 & 85 & 81 & 92 & 94 \\
\hline AM & $\operatorname{PPV}(\%)$ & 48 & 71 & 43 & 50 & 60 \\
\hline AM & NPV (\%) & 99 & 96 & 100 & 100 & 98 \\
\hline AM & Sensitivity (\%) & 92 & 92 & 100 & 100 & 75 \\
\hline AM & Specificity (\%) & 89 & 81 & 78 & 91 & 96 \\
\hline AM & Kappa & $0.58 * * * *$ & $0.68 * * *$ & $0.50 * * *$ & $0.63^{* * *}$ & $0.63^{* * * *}$ \\
\hline HR & Accuracy (\%) & 97 & 95 & 93 & 98 & 100 \\
\hline HR & PPV (\%) & 80 & 90 & 67 & 100 & 100 \\
\hline HR & NPV (\%) & 98 & 97 & 97 & 98 & 100 \\
\hline HR & Sensitivity (\%) & 80 & 90 & 80 & 50 & 100 \\
\hline HR & Specificity (\%) & 98 & 97 & 95 & 100 & 100 \\
\hline HR & Карpa & $0.78 * * *$ & $0.87^{* * * *}$ & $0.69 * * *$ & $0.66 * * *$ & $1.00^{* * * *}$ \\
\hline
\end{tabular}

Accuracy, positive (PPV) and negative (NPV) predictive values, sensitivity, specificity and Kappa of RTP-ASE using SPECT as method of reference. $C A$, coronary area; LAD, left anterior descending coronary artery; LCx, left circumflex artery; RPD, right posterior descending coronary artery, ${ }^{* * *}$ $=\mathrm{p}<0.001$.

analysis. Therefore, our results suggest that combined analysis is superior for maintaining good feasibility as well as good accuracy. It should be not that adenosine is not an optimal stressor concerning wall motion analysis. RTP-ASE is a highly available technique with possible bedside accessibility, which makes it appealing for clinical use, even in smaller hospitals with low access to angiographic or scintigraphic techniques.

\section{Study limitations}

One obvious limitation is the subjectivity of RTP-ASE, since it is based on visual estimation of both perfusion and wall motion. Software tools for quantification of perfusion have been developed, which have shown promising results mainly in animal models $[10,23,24]$ and a few clinical studies [16,25-29]. These results indicate that quantification of perfusion may soon be available for clin-

Table 4: Agreement between RTP-ASE angio-mode and high resolution.

$\begin{array}{ccccc}\text { Any CA } & \text { Patient } & \text { LAD } & \text { LCx } & \text { RPD } \\ (n=150) & (n=50) & (n=50) & (n=50) & (n=50)\end{array}$

$\begin{array}{lccccc}\text { RTP + WM } & & & & & \\ \text { Agreement (\%) } & 92 & 80 & 82 & 94 & 98 \\ \text { Kappa } & 0.61^{* * *} & 0.51^{* * *} & 0.43^{* *} & 0.64^{* * *} & 0.85^{* * *}\end{array}$

Sole RTP

$\begin{array}{llllll}\text { Agreement (\%) } & 88 & 77 & 78 & 91 & 94\end{array}$

\begin{tabular}{llllll} 
Kappa & $0.47^{* * *}$ & $0.52^{* *}$ & $0.43^{* *}$ & $0.37^{* *}$ & $0.54^{* * *}$ \\
\hline
\end{tabular}

Combined perfusion and wall motion analysis, and using solitary perfusion analysis.

CA, coronary area; LAD, left anterior descending coronary artery; $L C x$, left circumflex artery; RPD, right posterior descending coronary artery; RTP, real time perfusion; WM, wall motion; ** $=\mathrm{p}<0.01$; *** $=p<0.001$. ical use, but there is still need for larger clinical studies to evaluate feasibility. There are, however, need to improve user-friendly dedicated software, need for off-line analysis and the there is the additional cost of contrast agents.

It should be noted that this is an agreement study not an accuracy study. One limitation of the present investigation is that we cannot separate perfusion defects caused by macro-vascular or by micro-vascular disease because coronary angiography was not available in these patients. Further studies are needed to investigate the accuracy of RTP-ASE, SPECT, and perhaps Cardiac-MRI versus because coronary angiography.

Another limitation is that the RTP technique demands skilful operators and interpreters with a substantial amount of experience and knowledge in ultrasound physics, contrast agent characteristics and micro bubble behavior when exposed to ultrasound. However, this ultrasonic technique is still more available than other modalities and is useful for bedside evaluation, which still makes RTP-ASE an appealing and more accessible alternative.

The current study population consisted of patients with a high-risk for cardiovascular disease and this data cannot indiscriminately be used in low-risk patient-populations. Furthermore, the study results may obviously not be applicable to other types of populations with coronary artery disease, such as patients with acute coronary syndrome.

\section{Contrast safety}

The U.S. Food and Drug Administration issued on October the 10th 2007 a "black box" warning for perflutrencontaining contrast agents, which caused considerable 
Accuracy. RTP-ASE vs SPECT

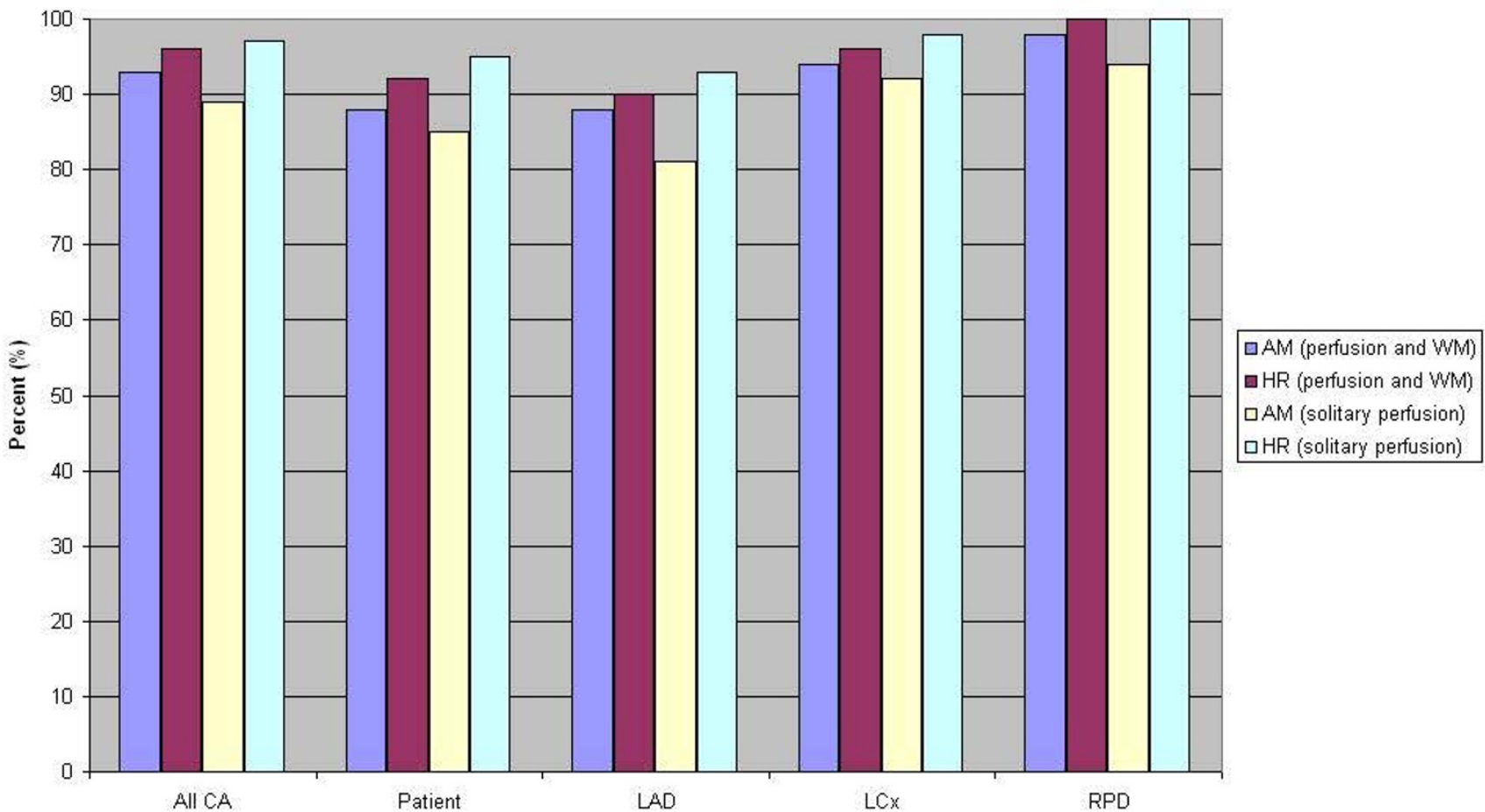

Figure 2

A graph of accuracy between different modalities of RTP-ASE versus SPECT. Left anterior descending (LAD), left circumflex (LCX) and right coronary artery (RCA). Angio-mode (AM), high resolution (HR) and wall motion (WM).

controversies within the echocardiography community [30]. This warning was later relaxed [31]. Three recent large retrospective studies have disputed the suggestion that using the current generation echo contrast would pose a hazard to the patient [31-33]. Kusnetzky and coworkers reported single-centre data on 18.671 consecutive studies and found no increased acute mortality in patients who had received a contrast agent [32]. Main et al. reported data from a multicenter registry that included 4.300.966 consecutive patients [31]. Their finding was that patients who had received echo contrast actually had a lower mortality rate compared to those who had not received contrast. Furthermore, Dolan et al. compared 23.659 patients from three U.S. medical centres who had received echo contrast, at a rest examination, with 5.900 controls who had not received contrast, and found no increased mortality or nonfatal myocardial infarct in patients who had received contrast [33]. Dolan and coworkers extended their analysis and compared 10.788 patients who had undergone stress echocardiography (DSE or exercise stress echocardiography) and received contrast with 15.989 who had not received contrast. No

Table 5: Non-interpretable coronary areas for RTP-ASE angio-mode (AM) and high resolution (HR).

\begin{tabular}{|c|c|c|c|c|c|c|}
\hline & & $\begin{array}{c}\text { Any CA } \\
(n=150)\end{array}$ & $\begin{array}{l}\text { Patient } \\
(n=50)\end{array}$ & $\begin{array}{c}\text { LAD } \\
(n=50)\end{array}$ & $\begin{array}{c}\text { LCx } \\
(n=50)\end{array}$ & $\begin{array}{c}\text { RPD } \\
(n=50)\end{array}$ \\
\hline AM & RTP + WM (\%) & 0 & 0 & 0 & 0 & 0 \\
\hline $\mathbf{A M}$ & Sole RTP (\%) & 13.3 & 22.0 & 16.0 & 24.0 & 0 \\
\hline HR & RTP + WM (\%) & 0 & 0 & 0 & 0 & 0 \\
\hline HR & Sole RTP (\%) & 10.7 & 22.0 & 12.0 & 16.0 & 4.0 \\
\hline
\end{tabular}

Combined perfusion and wall motion analysis, and solitary perfusion analysis.

CA, coronary area; LAD, left anterior descending coronary artery; LCx, left circumflex artery; RPD, right posterior descending coronary artery; RTP, real time perfusion; WM, wall motion. 
Table 6: Inter and intra observer agreement of myocardial contrast echocardiography ischemia interpretation.

\begin{tabular}{lcccc}
\hline & Total & LAD & LCx & RPD \\
\hline $\begin{array}{l}\text { Inter-observer (\%) AM } \\
\text { Kappa }\end{array}$ & $\begin{array}{c}91 \\
0.72^{* * *}\end{array}$ & $\begin{array}{c}88 \\
0.73^{* * * *}\end{array}$ & $\begin{array}{c}97 \\
0.90^{* * * *}\end{array}$ & $\begin{array}{c}88 \\
0.28 \mathrm{~ns}\end{array}$ \\
\hline $\begin{array}{l}\text { Intra-observer (\%) AM } \\
\text { Kappa }\end{array}$ & $\begin{array}{c}94 \\
0.76^{* * *}\end{array}$ & $\begin{array}{c}94 \\
0.84^{* * *}\end{array}$ & $\begin{array}{c}91 \\
0.62^{* * * *}\end{array}$ & $\begin{array}{c}97 \\
0.78^{* * * *}\end{array}$ \\
\hline $\begin{array}{l}\text { Inter-observer (\%) HR } \\
\text { Kappa }\end{array}$ & $\begin{array}{c}95 \\
0.70^{* * *}\end{array}$ & $\begin{array}{c}95 \\
0.64^{* * *}\end{array}$ & $\begin{array}{c}95 \\
0.64^{* * * *}\end{array}$ & $\begin{array}{c}95 \\
0.78^{* * * *}\end{array}$ \\
\hline $\begin{array}{l}\text { Intra-observer (\%) HR } \\
\text { Kappa }\end{array}$ & $\begin{array}{c}97 \\
0.78^{* * *}\end{array}$ & $\begin{array}{c}95 \\
0.64^{* *}\end{array}$ & $\begin{array}{c}95 \\
0.64\end{array}$ & $\begin{array}{c}100 \\
1.00^{* * *}\end{array}$ \\
\hline
\end{tabular}

RTP-ASE angio-mode (AM) and high resolution (HR) $(n=33)$.

LAD, Left anterior descending coronary artery; LCx, Left Circumflex artery; RPD, Right posterior descending coronary artery; ${ }^{* *}=p<$ $0.01 ; * * *=p<0.00 I$, ns $=$ not significant.

increased mortality or nonfatal myocardial infarct in patients who had received contrast could be found also in this cohort. These studies clearly show that using echo contrast in stable patients does not pose a significant risk. This knowledge must be weighted against the hazards of a non-diagnostic echocardiography examination, and the potential risks accompanied by alternative tests. Finally, cost effectiveness remains to be demonstrated and real additive value of routine use of echo contrast in every patient that undergo stress echocardiography.

\section{Conclusion}

The present investigation compared two different types RTP power modulation ASE. Both techniques showed highly significant agreements for the detection of reversible myocardial ischemia, using SPECT as method of reference. There was no significant difference between AM and HR in correctly detecting myocardial ischemia as judged by SPECT. This suggests that data derived from different techniques for RTP-ASE are reasonably interchangeable. When using the combined perfusion and wall motion interpretation, feasibility was higher, with almost no noninterpretable segments. Therefore, our results also suggest that combined analysis of perfusion and wall motion provides superior feasibility and accuracy of RTP-ASE.

\section{Competing interests}

The authors declare that they have no competing interests.

\section{Authors' contributions}

PG, RW, MD and RBW initiated the study. RW, MD and OT supervised the study and participated in the interpretation of the results and manuscript preparation. PG performed measurements, made all data conversions, plots and calculations from ultrasound data, and participated in the preparation of the manuscript. PG, KS, RW and MD participated in data collection, performed statistical analysis and participated in the interpretation of the results. LL and RBW participated in the interpretation of the results, in the creation of plots and in the preparation of the manuscript. MK participated in the interpretation of the results and preparation of the manuscript. All authors read and approved the final manuscript.

\section{Additional material}

\section{Additional file 1}

Movies of RTP-ASE images at rest on the left hand side, and stress images on the right hand side. Upper row represents angiomode modality and lower row represents grey scale images. Pleas note the apical lateral perfusion defect at stress.

Click here for file

[http://www.biomedcentral.com/content/supplementary/14767120-7-19-S1.avi]

\section{Acknowledgements}

This study was supported by grants from University Hospital UMAS, Malmö and The Laerdal Foundation for Acute Medicine. We thank the personnel at the Department of Clinical Physiology and the Department of Cardiology, University Hospital UMAS, Malmö for their skilful assistance with and in conjunction to scintigraphic and echocardiographic examinations.

\section{References}

I. Erhardt L, Herlitz J, Bossaert L, Halinen M, Keltai M, Koster R, Marcassa $C$, Quinn $T$, van Weert $H$ : Task force on the management of chest pain. Eur Heart / 2002, 23( I 5): I I 53-I I 76.

2. Cardiology TFotESo: Management of stable angina pectoris. Recommendations of the Task Force of the European Society of Cardiology. Eur Heart J 1997, 18(3):394-4I3.

3. Sicari R, Nihoyannopoulos P, Evangelista A, Kasprzak J, Lancellotti P, Poldermans D, Voigt JU, Zamorano JL: Stress echocardiography expert consensus statement: European Association of Echocardiography (EAE) (a registered branch of the ESC). Eur J Echocardiogr 2008, 9(4):415-437.

4. Underwood SR, Anagnostopoulos C, Cerqueira M, Ell PJ, Flint EJ, Harbinson M, Kelion AD, Al-Mohammad A, Prvulovich EM, Shaw L], et al.: Myocardial perfusion scintigraphy: the evidence. Eur J Nucl Med Mol Imaging 2004, 3 I (2):26I-29I.

5. Picano E: Stress echocardiography. Expert Rev Cardiovasc Ther 2004, 2(I):77-88.

6. Sozzi FB, Elhendy A, Roelandt JR, van Domburg RT, Schinkel AF, Vourvouri EC, Bax J, Rizzello V, Poldermans D: Long-term prognosis after normal dobutamine stress echocardiography. Am J Cardiol 2003, 92(II): 1267-I270.

7. Schinkel AF, Bax J], Geleijnse ML, Boersma E, Elhendy A, Roelandt JR, Poldermans $D$ : Noninvasive evaluation of ischaemic heart disease: myocardial perfusion imaging or stress echocardiography? Eur Heart J 2003, 24(9):789-800.

8. Takeishi Y, Chiba J, Abe S, Ikeda K, Tomoike H: Adenosineechocardiography for the detection of coronary artery disease. J Cardiol I994, 24(I): I-7.

9. Lafitte S, Matsugata H, Peters B, Togni M, Strachan M, Kwan OL, DeMaria AN: Comparative value of dobutamine and adenosine stress in the detection of coronary stenosis with myocardial contrast echocardiography. Circulation 2001, I 03(22):2724-2730.

10. Yao GH, Zhang C, Sun FR, Zhang M, Zhao YX, Zhang PF, Zhong L, Ding SF, Chen WQ, Li XN, et al:: Quantification of transmural gradient of blood flow in myocardial ischemia with real-time 
myocardial contrast echocardiography and dipyridamole stress test. Ultrasound Med Biol 2008, 34(I):22-30.

II. Winter R, Gudmundsson P, Willenheimer R: Real-time perfusion adenosine stress echocardiography in the coronary care unit: a feasible bedside tool for predicting coronary artery stenosis in patients with acute coronary syndrome. Eur J Echocardiogr 2005, 6(I):3I-40.

12. Sieswerda GT, Yang L, Boo MB, Kamp O: Real-time perfusion imaging: a new echocardiographic technique for simultaneous evaluation of myocardial perfusion and contraction. Echocardiography 2003, 20(6):545-555.

13. Olszowska M, Kostkiewicz M, Tracz W, Przewlocki T: Assessment of myocardial perfusion in patients with coronary artery disease. Comparison of myocardial contrast echocardiography and $99 \mathrm{mTC}$ MIBI single photon emission computed tomography. Int J Cardiol 2003, 90(I):49-55.

14. Mor-Avi V, Caiani EG, Collins KA, Korcarz CE, Bednarz JE, Lang RM: Combined assessment of myocardial perfusion and regional left ventricular function by analysis of contrast-enhanced power modulation images. Circulation 200I, 104(3):352-357.

15. Gudmundsson P, Winter R, Dencker M, Kitlinski M, Thorsson O, Ljunggren L, Willenheimer R: Real-time perfusion adenosine stress echocardiography versus myocardial perfusion adenosine scintigraphy for the detection of myocardial ischaemia in patients with stable coronary artery disease. Clin Physiol Funct Imaging 2006, 26(I):32-38.

16. Kowatsch I, Tsutsui JM, Osorio AF, Uchida AH, Machiori GG, Lopes ML, Cesar LA, Ramires JA, Mathias W Jr: Head-to-head comparison of dobutamine and adenosine stress real-time myocardial perfusion echocardiography for the detection of coronary artery disease. J Am Soc Echocardiogr 2007, 20(9): II09-III 7.

17. Lipiec $P$, Wejner-Mik P, Krzeminska-Pakula M, Kusmierek J, Plachcinska A, Szuminski R, Peruga JZ, Kasprzak JD: Accelerated stress real-time myocardial contrast echocardiography for the detection of coronary artery disease: comparison with $99 \mathrm{mTc}$ single photon emission computed tomography. J Am Soc Echocardiogr 2008, 2 I (8):941-947.

18. Wasmeier GH, Asmussen S, Voigt JU, Flachskampf FA, Daniel WG Nixdorff U: Real-time myocardial contrast stress echocardiography using bolus application. Ultrasound Med Biol 2008, 34(II): |724-I73I.

19. Becher H, Burns P: Handbook of Contrast Echocardiography. Left ventricular function and myocardial perfusion. Frankfurt and New York: Springer Verlag; 2000

20. Bahlmann EB, McQuillan BM, Handschumacher MD, Chow CM, Guerrero JL, Picard MH, Weyman AE, Scherrer-Crosbie M: Effect of destructive pulse duration on the detection of myocardial perfusion in myocardial contrast echocardiography: In vitro and in vivo observations. J Am Soc Echocardiogr 2002, I 5( I 2): | 440- | 447.

21. Henzlova MJ, Cerqueira MD, Mahmarian JJ, Yao SS: Stress protocols and tracers. J Nucl Cardiol 2006, I3(6):e80-90.

22. Hesse B, Tagil K, Cuocolo A, Anagnostopoulos C, Bardies M, Bax J Bengel F, Busemann Sokole E, Davies G, Dondi M, et al.: EANM/ESC procedural guidelines for myocardial perfusion imaging in nuclear cardiology. Eur J Nucl Med Mol Imaging 2005, 32(7):855-897.

23. Lafitte S, Higashiyama A, Masugata $\mathrm{H}$, Peters B, Strachan M, Kwan OL, DeMaria AN: Contrast echocardiography can assess risk area and infarct size during coronary occlusion and reperfusion: experimental validation. I Am Coll Cardiol 2002, 39(9): I546- 1554.

24. Agati L, Tonti G, Pedrizzetti G, Magri F, Funaro S, Madonna M, Celani $F$, Messager $T$, Broillet A: Clinical application of quantitative analysis in real-time MCE. Eur J Echocardiogr 2004, 5(Suppl 2):S17-23.

25. Bekeredjian R, Hilbel T, Filusch A, Hansen A, Benz A, Zehelein J, Kuecherer HF: Fourier phase and amplitude analysis for automated objective evaluation of myocardial contrast echocardiograms. Int / Cardiovasc Imaging 2003, 19(2): I 17- 28.

26. Peltier M, Vancraeynest D, Pasquet A, Ay T, Roelants V, D'Hondt AM, Melin JA, Vanoverschelde JL: Assessment of the physiologic significance of coronary disease with dipyridamole real-time myocardial contrast echocardiography. Comparison with technetium-99m sestamibi single-photon emission com- puted tomography and quantitative coronary angiography. Am Coll Cardiol 2004, 43(2):257-264.

27. Korosoglou G, da Silva KG Jr, Labadze N, Dubart AE, Hansen A, Rosenberg M, Zehelein J, Kuecherer $\mathrm{H}$ : Real-time myocardial contrast echocardiography for pharmacologic stress testing: is quantitative estimation of myocardial blood flow reserve necessary? J Am Soc Echocardiogr 2004, I 7(I): I-9.

28. Vogel R, Indermuhle A, Reinhardt J, Meier P, Siegrist PT, Namdar M, Kaufmann PA, Seiler C: The quantification of absolute myocardial perfusion in humans by contrast echocardiography: algorithm and validation. J Am Coll Cardiol 2005, 45(5):754-762.

29. Bierig SM, Mikolajczak P, Herrmann SC, Elmore N, Kern M, Labovitz AJ: Comparison of myocardial contrast echocardiography derived myocardial perfusion reserve with invasive determination of coronary flow reserve. Eur J Echocardiogr 2008.

30. Main ML, Goldman JH, Grayburn PA: Thinking outside the "box"the ultrasound contrast controversy. I Am Coll Cardiol 2007, 50(25):2434-2437.

31. Main ML, Ryan AC, Davis TE, Albano MP, Kusnetzky LL, Hibberd M: Acute mortality in hospitalized patients undergoing echocardiography with and without an ultrasound contrast agent (multicenter registry results in 4,300,966 consecutive patients). Am J Cardiol 2008, I 02(I 2): 1742-1746.

32. Kusnetzky LL, Khalid A, Khumri TM, Moe TG, Jones PG, Main ML: Acute mortality in hospitalized patients undergoing echocardiography with and without an ultrasound contrast agent: results in 18,67I consecutive studies. J Am Coll Cardiol 2008, 5 I ( I 7): 1704-1706.

33. Dolan MS, Gala SS, Dodla S, Abdelmoneim SS, Xie F, Cloutier D, Bierig M, Mulvagh SL, Porter TR, Labovitz AJ: Safety and efficacy of commercially available ultrasound contrast agents for rest and stress echocardiography a multicenter experience. J Am Coll Cardiol 2009, 53(I):32-38.

Publish with Bio Med Central and every scientist can read your work free of charge

"BioMed Central will be the most significant development for disseminating the results of biomedical research in our lifetime. "

Sir Paul Nurse, Cancer Research UK

Your research papers will be:

- available free of charge to the entire biomedical community

- peer reviewed and published immediately upon acceptance

- cited in PubMed and archived on PubMed Central

- yours - you keep the copyright 